\title{
Detection of Pancreatic Cancer by Urine Volatile Organic Compound Analysis
}

\author{
SAMULI I. NISSINEN ${ }^{1,2}$, ANTTI ROINE ${ }^{3}$, LAURI HOKKINEN ${ }^{4}$, MARKUS KARJALAINEN $^{5}$, \\ MARKUS VENÄLÄINEN ${ }^{1}$, HELI HELMINEN ${ }^{6}$, RIIKKA NIEMI ${ }^{7}$, \\ TERHO LEHTIMÄKI ${ }^{4,8,9}$, TUOMO RANTANEN ${ }^{1,10^{*}}$ and NIKU OKSALA ${ }^{4,9,11^{*}}$ \\ ${ }^{1}$ School of Medicine, University of Eastern Finland, Kuopio, Finland; \\ ${ }^{2}$ Department of Gastroenterology, Kuopio University Hospital, Kuopio, Finland; \\ ${ }^{3}$ Department of Surgery, Hatanpää Hospital, Tampere, Finland; \\ ${ }^{4}$ Faculty of Medicine and Health Technology, Tampere University, Tampere, Finland; \\ ${ }^{5}$ BioMediTech Institute and Faculty of Biomedical Sciences and Engineering, \\ Tampere University of Technology, Tampere, Finland; \\ ${ }^{6}$ Seinäjoki Central Hospital, Seinäjoki, Finland; \\ ${ }^{7}$ Department of Obstetrics and Gynecology, Tampere University Hospital, Tampere, Finland; \\ ${ }^{8}$ Department of Clinical Chemistry, Fimlab Laboratories, Tampere, Finland; \\ ${ }^{9}$ Finnish Cardiovascular Research Center Tampere, Tampere, Finland; \\ ${ }^{10}$ Department of Surgery, Kuopio University Hospital, Kuopio, Finland; \\ ${ }^{11}$ Department of Vascular Surgery, Tampere University Hospital, Tampere, Finland
}

\begin{abstract}
Background/Aim: Most pancreatic cancer patients are diagnosed at an advanced stage, since the diagnosis is demanding. Field asymmetric waveform ion mobility spectrometry (FAIMS) is a sensitive technique used for the detection of volatile organic compounds (VOC). We evaluated the ability of FAIMS to discriminate between pancreatic cancer and healthy controls from a urine sample. Patients and Methods: For a proof-of-concept study in three Finnish hospitals, 68 patients with pancreatic cancer, 36 with acute pancreatitis, 18 with chronic pancreatitis, 8 with pancreatic pre-malign lesions and 52 healthy controls were prospectively recruited. Urine samples were collected at the time of diagnosis and stored at $-70^{\circ} \mathrm{C}$. The samples were subsequently measured with FAIMS. The data were processed with linear discriminant analysis and crossvalidated with leave-one-out cross-validation. Results:
\end{abstract}

This article is freely accessible online.

*These Authors contributed equally to this study.

Correspondence to: Samuli I. Nissinen, Eastern Finland University, Kuopio, Finland, Kanta-Häme Central Hospital, Ahvenistontie 20, Hämeenlinna 13530, Finland. Tel: +358 443062131, e-mail: samuli.nissinen@gmail.com

Key Words: Pancreatic cancer, pre-malignant lesion, urine test, FAIMS, volatile organic compound, linear discriminant analysis.
FAIMS distinguished pancreatic cancer from controls with a sensitivity of $79 \%$ and specificity of $79 \%$. Conclusion: As a non-invasive and rapid urine test, FAIMS can discriminate patients with pancreatic cancer from healthy controls.

Pancreatic cancer is the eleventh most common cancer globally, but due to its poor prognosis it is the fourth leading cause of cancer death in Western countries (1-3). The mortality rates of all common cancers are predicted to decline, with the exception of pancreatic cancer $(4,5)$. The prognosis for pancreatic cancer patients has remained poor for the last four decades, but reports from recent years have shown modest improvements $(1,6)$. If pancreatic cancer is found at an early stage, it would be possible to achieve a much better prognosis (7-9). To date, a method to screen the entire population does not exist, but some studies have shown promising results in detecting pancreatic cancer in high-risk populations. Mayerle et al. achieved an excellent sensitivity and specificity in differentiating pancreatic cancer from chronic pancreatitis using metabolic biomarker testing combined with CA19-9 (10).

The relatively low incidence of pancreatic cancer combined with its very high mortality rate makes it very difficult to find any applicable screening method (8). As has been demonstrated with colon cancer, the best hope for reducing cancer-specific mortality lies in early diagnosis and treatment, ideally at a precancerous stage (11). The major pancreatic pre-malign lesions are intraductal papillary 
mucinous neoplasm (IPMN) and mucinous cystic neoplasms $(\mathrm{MCN})$, which are present for years before they progress to invasive cancer (12-14). Therefore, they should be detected as IPMN or MCN before they advance into pancreatic cancers.

Recently, the first article about the use of field asymmetric ion mobility spectrometry (FAIMS) analysis of volatile organic compounds (VOC) from urine headspace, distinguishing pancreatic cancer and control patients was published (15). In that study, however, pancreatic pre-malign lesions or pancreatitis were not analysed. Previous reports have demonstrated the capability of FAIMS and an electronic nose in the detection of different origin cancer from a urine sample (16-19).

The aim of this study was to study whether urine FAIMS analysis could be used to discriminate between pancreatic cancer, pancreatic pre-malign lesions and healthy controls. The second aim was to study whether FAIMS could discriminate acute and chronic pancreatitis from controls and pancreatic cancer.

\section{Patients and Methods}

Patients. All patients were prospectively enrolled in this study at three Finnish hospitals, the Seinäjoki Central Hospital, Tampere University Hospital and Kuopio University Hospital, between October 2013 and June 2016. Written informed consent was acquired from all subjects. This study was approved by the ethical committee of Tampere University Hospital (code: R10066). The study protocol conforms to the ethical guidelines of the 1975 Declaration of Helsinki as reflected in a priori approval by the institution's human research committee.

The inclusion criteria for study subjects were a new diagnosis of pancreatic cancer or a pancreatic pre-malign lesion, or acute pancreatitis or chronic pancreatitis at the emergency unit. The controls comprised otherwise healthy patients with no known cancer history scheduled for a hernia operation, elective cholecystectomy or pelvic organ prolapse operation. The controls were enrolled during the same period as the pancreatic cancer patients, and they gave a urine sample on the morning of the operation day. The exclusion criteria were failures in sample preparation or incorrect diagnosis in follow-up.

Pancreatic cancer was diagnosed with computer tomography (CT), magnetic resonance imaging (MRI) or endoscopic ultrasound (EUS). The diagnosis of pancreatic ductal adenocarcinoma was later confirmed with a biopsy and histology analysis by a pathologist. The diagnoses of pre-malign pancreatic tumours were made after tumour biopsy and histology or cytology, including IPMN, adenoma with high-grade dysplasia and MCN with high-grade dysplasia, but with no sign of pancreatic adenocarcinoma.

Information on disease status during the follow-up was collected from the patients' hospital records from the time of study enrolment until 6 April 2017. The diagnoses of all enrolled patients who survived were confirmed after a median of 1.9 years' follow-up (range $=0.8-2.9$ years). Uncertain diagnoses for patients who died were confirmed from autopsy documents. The vital statuses were checked from the Finnish Population Register Centre.
Table I. Clinical characteristics of patients and controls.

\begin{tabular}{lccccc}
\hline Characteristics & PDAC & AP & CP & PLP & Controls \\
\hline $\mathrm{n}$ & 68 & 36 & 18 & 8 & 52 \\
Male & $36 / 53 \%$ & $24 / 67 \%$ & $14 / 78 \%$ & $5 / 63 \%$ & $24 / 46 \%$ \\
Female & 32 & 12 & 4 & 3 & 28 \\
Age, years & & & & & \\
$\quad$ Median & 71 & 62 & 58 & 66 & 58 \\
IQR & $64-77$ & $48-72$ & $55-65$ & $58-71$ & $51-69$ \\
Stage & & & & & \\
IA & 0 & & & & \\
IB & 8 & & & & \\
IIA & 0 & & & & \\
IIB & 11 & & & & \\
III & 21 & & & & \\
IV & 28 & & & & \\
Non-operable & 50 & & & \\
\hline
\end{tabular}

AP: Acute pancreatitis; CP: chronic pancreatitis; PDAC: pancreatic adenocarcinoma; PLP: pre-malign lesion of the pancreas.

Urine sample preparation. The day after the diagnosis, the patients provided $100 \mathrm{ml}$ urine samples with a bladder time of at least 4 hours. The samples were stored at $-70^{\circ} \mathrm{C}$ until analysis. The urine samples were collected prospectively without preservatives. All patients were on unreported self-selected diets. The controls provided morning urine samples before their operation.

Before analysis, the urine specimens were thawed unassisted at room temperature and mixed with a vortex mixer. For the analysis, $5 \mathrm{ml}$ of urine were pipetted into a $30 \mathrm{ml}$ glass vial. The rest of the urine was pipetted and stored into $5 \mathrm{ml}$ tubes for later use. The urine specimens were then left to warm at room temperature for at least one hour before analysis.

Field asymmetric ion-mobility spectrometry (FAIMS). For the FAIMS analysis, we utilised a Lonestar chemical analyzer (Owlstone, UK), employing an ATLAS sampling system and a split flow box. This system achieves the separation of chemical components on the basis of differences in the electric field dependence of ionised chemical mobilities. In contrast to traditional analytic techniques such as mass spectrometry, FAIMS allows gas molecules to be separated and analysed at atmospheric pressure and room temperature, resulting in significantly reduced complexity and cost of the device. After a sample is ionized, it is composed with ions of various sizes and masses. These are introduced between two metal plates, and an asymmetric high-voltage waveform is applied to these plates, subjecting the ionized molecules to high and low electric fields in an intermittent manner. The characteristics of the molecules result in different velocities in the movement of these molecules within the high- and low-electric field conditions, which can be measured, resulting in a separation of the complex mixture. We used the same settings as described by Arasaradnam et al. (15). In brief, the prepared sample was placed in the ATLAS sampling unit, which was warmed to $40^{\circ} \mathrm{C}$. Clean synthetic air was used as a carrier gas. The flow rate over the sample was $500 \mathrm{ml} / \mathrm{min}$, and the flow was mixed to $2,000 \mathrm{ml} / \mathrm{min}$ of clean air for a total of $2,500 \mathrm{ml} / \mathrm{h}$, which was passed into the instrument. The filter temperature was $70^{\circ} \mathrm{C}$ and the 
Table II. Results; FAIMS signal data LDA classification.

\begin{tabular}{lcccc}
\hline Classification pairs & Right positive & Right negative & Sensitivity (95\%CI) & Specificity (95\%CI) \\
\hline PDAC $v s$. controls & $54 / 68$ & $41 / 52$ & $0.79(0.68-0.87)$ & $0.79(0.66-0.88)$ \\
PDAC + PLP $v s$. controls & $64 / 76$ & $39 / 52$ & $0.85(0.74-0.91)$ & $0.75(0.62-0.85)$ \\
AP $v s$. controls & $24 / 36$ & $43 / 52$ & $0.67(0.53-0.78)$ & $0.83(0.70-0.91)$ \\
CP $v s$. controls & $8 / 18$ & $47 / 52$ & $0.44(0.25-0.66)$ & $0.9(0.79-0.96)$ \\
PDAC + PLP + AP + CP $v s$. controls & $117 / 129$ & $21 / 45$ & $0.91(0.84-0.95)$ & $0.47(0.33-0.61)$ \\
\hline
\end{tabular}

AP: Acute pancreatitis; CP: chronic pancreatitis; PLP: pre-malign lesion of the pancreas; PDAC: pancreatic adenocarcinoma.

lid temperature $120^{\circ} \mathrm{C}$. The FAIMS was set up to scan a $0-90 \%$ dispersion field in 51 steps and a compensation voltage of between $-6 \mathrm{~V}$ and $+6 \mathrm{~V}$ in 512 steps. Each sample took $30 \mathrm{sec}$ to scan. We repeated three scans for each sample. We scanned 20 samples per hour and 40-100 samples per day. We ran three scans with a sterilized water sample between each urine sample scan for cleaning purposes. To reduce carry-over effects, the FAIMS system was left overnight in continuous scanning mode with a sterilized water sample between runs.

Statistical analysis. For each urine sample, the data from the third scan were used for analysis. Each FAIMS matrix consisted of 52,200 measuring points per sample. These measurement points were condensed to 3,200 per sample to significantly speed up the analysis. Therefore, in the analysis, the final data of each sample were 3,200-dimensional. The analysis was conducted with MATLAB R2016b (Mathworks Inc, Natick, MA, USA). Linear discriminant analysis (LDA) was used to create classification parameters. In LDA, the data points are projected to a subspace in which the different classes of the original data are the most distinguishable from each other. To avoid over-fitting, the results were cross validated using leave-one-out cross validation (LOOCV). In this method, one by one, each sample was first removed from the data pool, and the classification parameters were then created using all the remaining samples as the training set. The single removed sample, acting as the test set, was then classified using these parameters.

\section{Results}

The characteristics of the study patients and controls are presented in Table I. Overall, 82 patients with suspected pancreatic cancer were recruited. After a total of 2.9 years of follow-up, 14 patients were excluded. The causes for the exclusions were as follows: diagnoses of cholangiocarcinoma ( 2 patients), metastasis in the pancreas (1 patient), IPMN (1 patient was moved to the pre-malign lesions group), pancreatic abscess ( 1 patient), acute pancreatitis (1 patient was moved to the acute pancreatitis group), misdiagnosis of pancreatic cancer (1 patient), and sampling failure (7 patients). Finally, 68 patients had a confirmed diagnosis with pancreatic ductal adenocarcinoma and included in the pancreatic cancer group. Out of the pancreatic cancer patients, $74 \%$ were inoperable, $28 \%$ had stage IB-IIB and $72 \%$ stage III-IV pancreatic cancer, and $26 \%$ underwent radical pancreatoduodenectomy or caudal resection. The main results are presented in Table II. The FAIMS signal data distinguished pancreatic cancer patients and controls with a sensitivity of $79 \%(95 \% \mathrm{CI}=0.68-0.87)$ and a specificity of $79 \%(95 \% \mathrm{CI}=0.66-0.88)$. FAIMS had the ability to detect pancreatic cancer and pancreatic pre-malign lesions $(n=68+8)$ with a sensitivity of $85 \%(95 \% \mathrm{CI}=0.74-0.91)$ and specificity of $75 \%(95 \% \mathrm{CI}=0.62-0.85)$. FAIMS discriminated between acute pancreatitis and controls with a sensitivity of $67 \%$ (95\% CI $=0.50-0.80)$ and specificity of $83 \%(95 \% \mathrm{CI}=0.70-$ $0.91)$. FAIMS could not differentiate pancreatic cancer from either acute or chronic pancreatitis nor chronic pancreatitis from controls. We also compared all of the pancreatic diseases - cancer, pre-malign lesions and pancreatitis - with controls. The analysis showed a sensitivity of $91 \%$ $(95 \% \mathrm{CI}=0.84-0.95)$ and a specificity of $47 \%(95 \% \mathrm{CI}=0.33-$ $0.61)$. The representative 'plumes' produced by FAIMS analysis plotted on a log scale are shown in Figure 1. Plots A and $\mathrm{B}$ represent typical FAIMS signal data concerning pancreatic cancer in a graphic spectrum mode. Plots $C$ and $D$ are representative spectrums of a control urine sample. Furthermore, plots $\mathrm{E}$ and $\mathrm{F}$ are representative spectrums of acute pancreatitis.

\section{Discussion}

The main finding of this study was that FAIMS can distinguish pancreatic cancer and pancreatic pre-malign lesions from healthy controls based on a urine specimen. The secondary finding was that FAIMS discriminates acute pancreatitis from controls, but the sensitivity was lower than with pancreatic cancer.

In microRNA (miRNA) studies, it has been shown that the presence of pancreatic cancer in the body is reflected in urine and that urine is a useful biofluid in the detection of pancreatic cancer (20). With similar technology and slightly different measuring methods, Arasaradnam et al. were able to distinguish 81 pancreatic cancer patients from 81 control 
patients using FAIMS analysis of urine with a sensitivity of 0.91 (95\% CI=0.83-0.96) and a specificity of 0.83 $(95 \% \mathrm{CI}=0.73-0.90)(15)$. Our results are in line with those findings and further support the use of FAIMS in the diagnosis of pancreatic cancer. A significant difference that also explains our slightly inferior results is that we used a cross-validation method, which results in more realistic, more likely reproducible and generalizable results. Another difference potentially explaining the differences was that non-malignant cases, i.e. patients with acute and chronic pancreatitis and premalign pancreatic lesions, were also included in our analyses. Also, a slightly different measuring protocol was used. We measured approximately 20 samples per h, which amounts to an average of 3 min per sample, while Arasaradnam et al. placed the vial with the urine sample in the ATLAS sampling system at $40^{\circ} \mathrm{C}$ for 10 min before measuring it, resulting in an average maximum of 5 samples per $h$.

As a novel finding, we showed that FAIMS can discriminate pancreatic cancer and pre-malign pancreatic lesions from controls. In addition, we found that acute pancreatitis can be discriminated from the controls, although the sensitivity was not as high as in detecting pancreatic cancer. FAIMS could discriminate pancreatic diseases - pancreatic cancer, pre-malign lesions and pancreatitis - as a single group from the controls, but the specificity was poor. Unfortunately, FAIMS could not discriminate acute and chronic pancreatitis from pancreatic cancer and cannot therefore be used in the discrimination of chronic pancreatitis from pancreatic cancer as such. This is also, to the best of our knowledge, reported herein for the first time. It can therefore be speculated that the VOC profile of pancreatic cancer is somehow related to the pancreatic inflammatory response, which may be a common feature of pancreatitis and pancreatic cancer, a hypothesis supported by the previous findings of chronic pancreatitis as a significant risk factor for the development of pancreatic cancer (21-23).

Trained dogs can detect different-origin cancers from a urine sample with much better sensitivity, specificity and positive predictive value than any man-made systems, demonstrating proof of the concept that cancer has a specific smell-print, and cancer-specific chemical compounds may therefore be circulating systemically throughout the body and secreted into urine $(24,25)$. The best detecting accuracy from urine samples has been shown with prostatic cancer (26). We did not find any studies with dogs and pancreatic cancer. Although dogs can detect cancers, their use in clinical screening is impossible because of the low prevalence of cancer in a screening population. It is known from experience regarding canine olfactory professional use that dogs can accurately detect differences between only a few samples at a time and then they need a resting period (27).

The potential of FAIMS lies in its high sensitivity for the trace concentrations of molecules. The measurement can be tailored for molecules with specific mobility by adjusting the parameters of the asymmetric high-voltage electric field. On the contrary, the whole molecular spectrum of the sample can be qualitatively measured from a single urine sample and within a single measurement, instead of one or several special markers or a combination of markers (28-30). FAIMS technology has also been shown to be accurate in quantitative analysis, especially when used together with mass spectrometry. Our approach was, analogously to canine olfaction, qualitative, with the aim of finding features that discriminate samples. The specific compounds that discriminate cancerous and noncancerous patients are currently unclear, with some studies pointing to molecules that reflect oxidative stress (31). We have recently reported about elevated concentrations of polyamines, organ molecules with strong scent in the urine of colorectal cancer patients (32). Polyamine metabolism have important role in pancreatic ductal adenocarcinoma (33). We hypothesize that polyamines may play a significant role in the "smell of cancer".

The first limitation of our study is missing samples. A total of 82 patients with a diagnosis of pancreatic cancer were recruited. After attaining the histology of patients with suspected pancreatic cancer and after a careful follow-up, seven patients were excluded because of different diagnosis than pancreatic cancer. Unfortunately, there were also seven patients with a diagnosis of pancreatic cancer who did not provide a urine sample or whose sample was not sent to the laboratory. This was a problem in our recruiting protocol. Many samples were taken at the emergency unit or on the patient's first day in hospital immediately after a positive CT scan for pancreatic cancer. We could not guarantee the delivery of all urine samples to the laboratory. The storage time at $-70^{\circ} \mathrm{C}$ varied due to the prospective sample collection and single laboratory period. There is limited evidence that storage time and aging affect VOC profiles, which can hinder the discrimination rate in this study (34). Therefore, urine preservation might also be a research target.

Ghatnekar et al. argued that a cost-effective pancreatic cancer screening test should exceed $88 \%$ sensitivity and $85 \%$ specificity when the cost per test is $€ 400$ and the pancreatic cancer incidence in the screening population is as high as that of newly diagnosed diabetics among elderly individuals $(0.71 \%$ per year three years after diagnosis $(35,36)$. These criteria can be relaxed when the test is more inexpensive or the incidence rises as in hereditary pancreatic cancer (37). In our study, FAIMS did not reach this accuracy. The measurement system needs to be further developed, and more research is needed to increase the accuracy of FAIMS to exceed $90 \%$, a screening threshold for a population at high risk of developing pancreatic cancer.

To the best of our knowledge, it was shown, for the first time, that there is a difference in urine volatile compounds between patients with pancreatic cancer or a pre-malign pancreatic lesion and controls. In addition, FAIMS could discriminate acute pancreatitis from controls. 
A

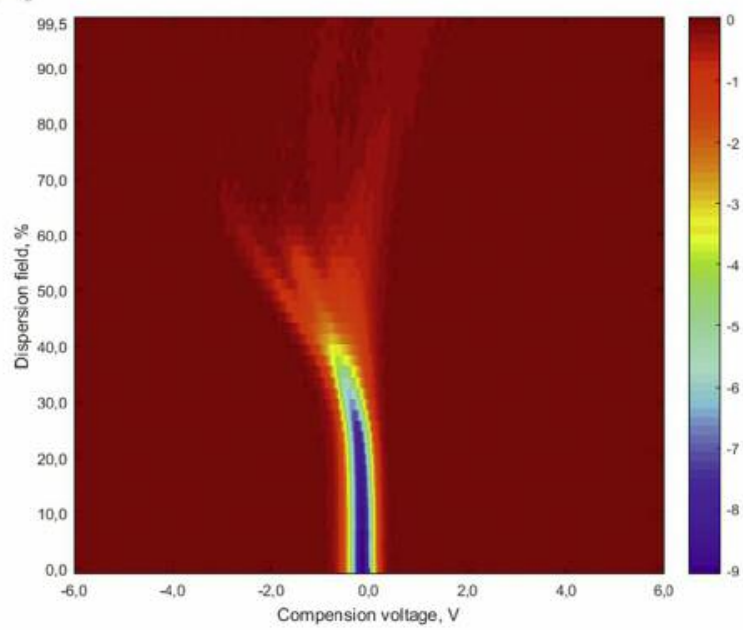

C

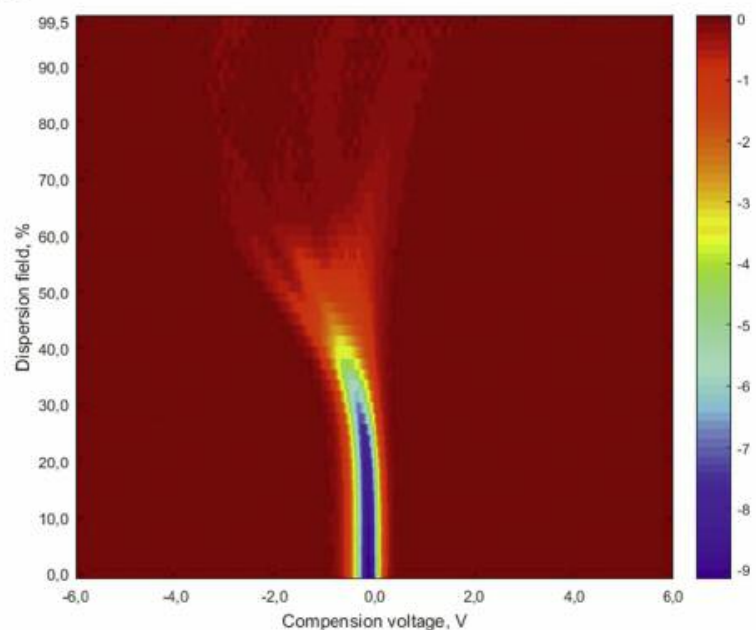

E

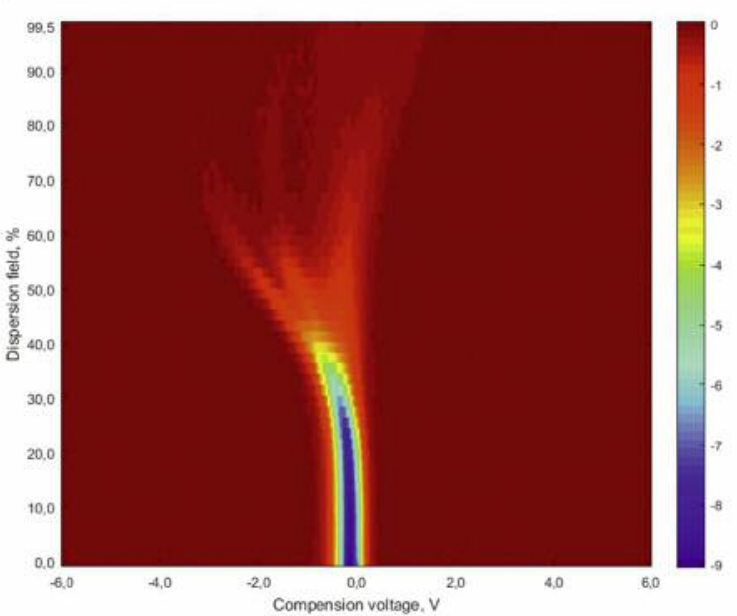

B

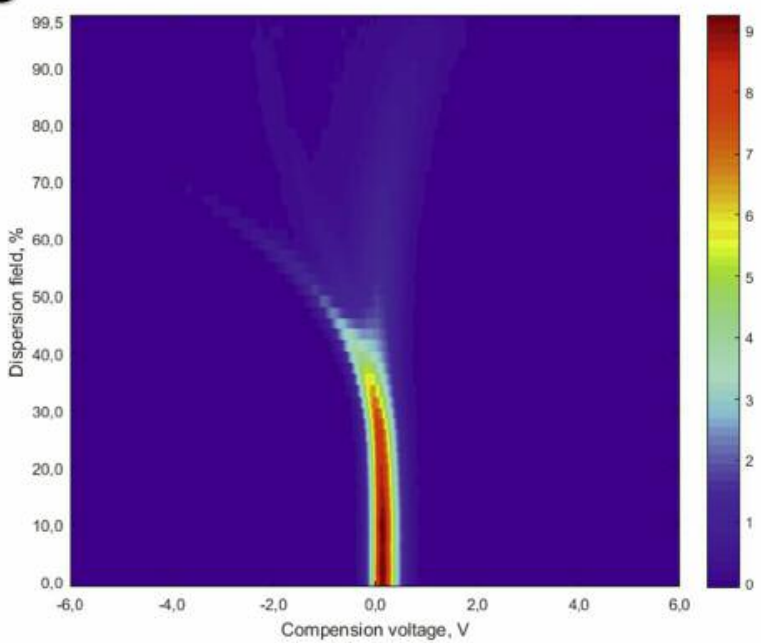

D

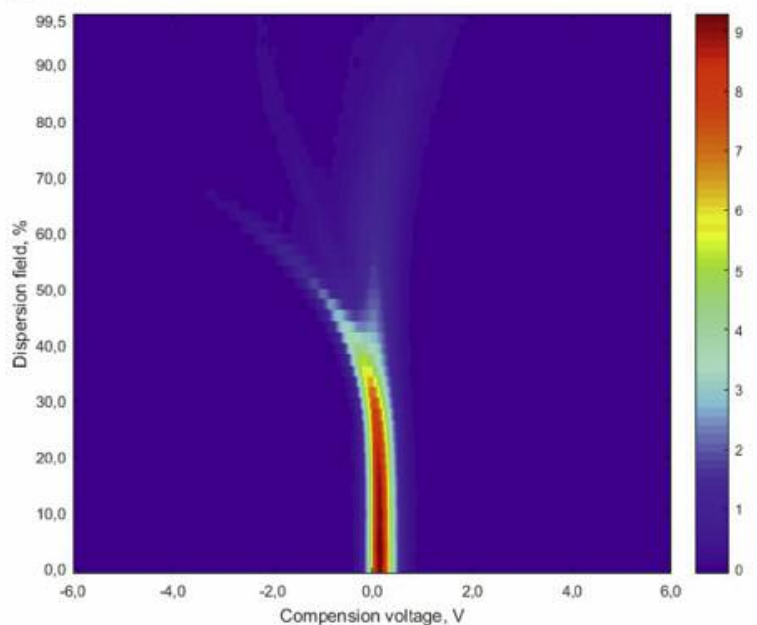

F

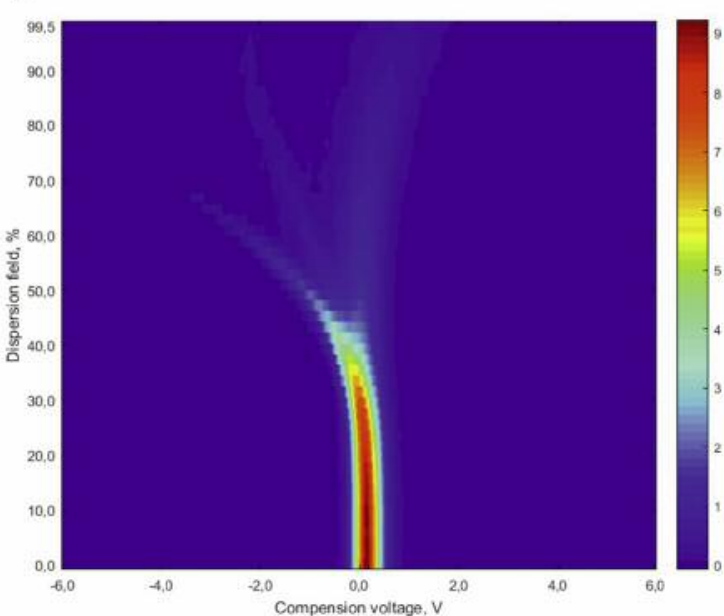

Figure 1. FAIMS spectrums of a pancreatic cancer and a control sample. Negative (A) and positive (B) panels of compensation voltage of a representative pancreatic cancer sample. Negative $(C)$ and positive $(D)$ compensation voltage spectrums of a control sample. Negative $(E)$ and positive $(F)$ compensation voltage spectrums of an acute pancreatitis sample. 


\section{Conflicts of Interest}

Niku Oksala, Antti Roine and Markus Karjalainen are shareholders of Olfactomics Ltd, a company developing a device for intraoperative tissue assessment with eNose -technology.

\section{Acknowledgements}

The Authors would like to thank Jeffrey Ahern and Katrina Ahern for English language editing and Ari Palomaki and Markku Heikkinen for critically reading the manuscript. This study was supported by grants from the following foundations: Finnish State Research Funding, Kuopio University Hospital Research Funding, Pirkanmaa Hospital District Research Funding, grants no. 1V285/5200635, no. 13107/5200635 9s045, 151B03, 9T044, 9U042, 150618 and 9V044.

\section{References}

1 Sirri E, Castro FA, Kieschke J, Jansen L, Emrich K, Gondos A, Holleczek B, Katalinic A, Urbschat I, Vohmann C and Brenner $\mathrm{H}$ : Recent trends in survival of patients with pancreatic cancer in Germany and the United States. Pancreas 45: 908-914, 2016.

2 Ilic $\mathrm{M}$ and Ilic I: Epidemiology of pancreatic cancer. World J Gastroenterol 22: 9694-9705, 2016.

3 Carrato A, Falcone A, Ducreux M, Valle JW, Parnaby A, Djazouli K, Alnwick-Allu K, Hutchings A, Palaska C and Parthenaki I: A Systematic review of the burden of pancreatic cancer in europe: real-world impact on survival, quality of life and costs. J Gastrointest Cancer 46: 201-211, 2015.

4 Malvezzi M, Carioli G, Bertuccio P, Boffetta P, Levi F, La Vecchia $\mathrm{C}$ and Negri E: European cancer mortality predictions for the year 2017, with focus on lung cancer. Ann Oncol 28: 1117-1123, 2017.

5 Ferlay J, Partensky C and Bray F: More deaths from pancreatic cancer than breast cancer in the EU by 2017. Acta Oncol 55: 1158-1160, 2016.

6 Karakas Y, Lacin S and Yalcin S: Recent advances in the management of pancreatic adenocarcinoma. Expert Rev Anticancer Ther 18: 51-62, 2018.

7 Egawa S, Takeda K, Fukuyama S, Motoi F, Sunamura M and Matsuno S: Clinicopathological aspects of small pancreatic cancer. Pancreas 28: 235-240, 2004.

8 Kim VM and Ahuja N: Early detection of pancreatic cancer. Chin J Cancer Res 27: 321-331, 2015.

9 Shimizu Y, Yasui K, Matsueda K, Yanagisawa A and Yamao K: Small carcinoma of the pancreas is curable: New computed tomography finding, pathological study and postoperative results from a single institute. J Gastroenterol Hepatol 20: 1591-1594, 2005.

10 Mayerle J, Kalthoff H, Reszka R, Kamlage B, Peter E, Schniewind B, González Maldonado S, Pilarsky C, Heidecke CD, Schatz P, Distler M, Scheiber JA, Mahajan UM, Weiss FU, Grützmann R and Lerch MM: Metabolic biomarker signature to differentiate pancreatic ductal adenocarcinoma from chronic pancreatitis. Gut 67: 128-137, 2018.

11 Zauber AG, Winawer SJ, O'Brien MJ, Lansdorp-Vogelaar I, van Ballegooijen M, Hankey BF, Shi W, Bond JH, Schapiro M, Panish JF, Stewart ET and Waye JD: Colonoscopic polypectomy and long-term prevention of colorectal-cancer deaths. N Engl J Med 366: 687-696, 2012.
12 Brune K, Abe T, Canto M, O’Malley L, Klein AP, Maitra A, Volkan Adsay N, Fishman EK, Cameron JL, Yeo CJ, Kern SE, Goggins $\mathrm{M}$ and Hruban RH: Multifocal neoplastic precursor lesions associated with lobular atrophy of the pancreas in patients having a strong family history of pancreatic cancer. Am J Surg Pathol 30: 1067-1076, 2006.

13 Feldmann G, Beaty R, Hruban RH and Maitra A: Molecular genetics of pancreatic intraepithelial neoplasia. J Hepatobiliary Pancreat Surg 14: 224-232, 2007.

14 Kanda M, Matthaei H, Wu J, Hong S-M, Yu J, Borges M, Hruban RH, Maitra A, Kinzler K, Vogelstein B and Goggins M: Presence of somatic mutations in most early-stage pancreatic intraepithelial neoplasia. Gastroenterology 142: 730-733.e9, 2012.

15 Arasaradnam R, Wicaksono A, O'Brien H, Kocher HM, Covington JA and Crnogorac-Jurcevic T: Non-invasive diagnosis of pancreatic cancer through detection of volatile organic compounds in urine. Gastroenterology 154: 485-487.e1, 2018.

16 Arasaradnam RP, McFarlane MJ, Ryan-Fisher C, Westenbrink E, Hodges P, Hodges P, Thomas MG, Chambers S, O'Connell N, Bailey C, Harmston C, Nwokolo CU, Bardhan KD and Covington JA: Detection of colorectal cancer (CRC) by urinary volatile organic compound analysis. PloS one 9: e108750, 2014.

17 Bernabei M, Pennazza G, Santonico M, Corsi C, Roscioni C, Paolesse R, Di Natale C and D'Amico A: A preliminary study on the possibility to diagnose urinary tract cancers by an electronic nose. Sensors and Actuators B: Chemical 131: 1-4, 2008.

18 Silva CL, Passos M and Câmara JS: Investigation of urinary volatile organic metabolites as potential cancer biomarkers by solid-phase microextraction in combination with gas chromatography-mass spectrometry. Br J Cancer 105: 18941904, 2011.

19 Roine A, Veskimäe E, Tuokko A, Kumpulainen P, Koskimäki J, Keinänen TA, Häkkinen MR, Vepsäläinen J, Paavonen T, Lekkala J, Lehtimäki T, Tammela TL and Oksala NKJ: Detection of prostate cancer by an electronic nose: a proof of principle study. J Urol 192: 230-234, 2014.

20 Debernardi S, Massat NJ, Radon TP, Sangaralingam A, Banissi A, Ennis DP, Dowe T, Chelala C, Pereira SP, Kocher HM, Young BD, Bond-Smith G, Hutchins R and Crnogorac-Jurcevic T: Noninvasive urinary miRNA biomarkers for early detection of pancreatic adenocarcinoma. Am J Cancer Res 5: 3455-3466, 2015.

21 Lowenfels AB, Maisonneuve P, Cavallini G, Ammann RW, Lankisch PG, Andersen JR, Dimagno EP, Andren-Sandberg A, Domellof L and Group the IPS: Pancreatitis and the risk of pancreatic cancer. NEngl J Med 328: 1433-1437, 1993.

22 Malka D, Hammel P, Maire F, Rufat P, Madeira I, Pessione F, Lévy P and Ruszniewski P: Risk of pancreatic adenocarcinoma in chronic pancreatitis. Gut 51: 849-852, 2002.

23 Whitcomb DC: Inflammation and Cancer V. Chronic pancreatitis and pancreatic cancer. Am J Physiol Gastrointest Liver Physiol 287: G315-G319, 2004.

24 Lippi G and Cervellin G: Canine olfactory detection of cancer versus laboratory testing: myth or opportunity? Clin Chem Lab Med 50: 435-439, 2012.

25 Leitch O, Anderson A, Paul Kirkbride K and Lennard C: Biological organisms as volatile compound detectors: A review. Forensic Sci Int 232: 92-103, 2013. 
26 Taverna G, Tidu L, Grizzi F, Torri V, Mandressi A, Sardella P, La Torre G, Cocciolone G, Seveso M, Giusti G, Hurle R, Santoro A and Graziotti P: Olfactory system of highly trained dogs detects prostate cancer in urine samples. J Urol 193: 13821387, 2015.

27 Edwards TL, Browne CM, Schoon A, Cox C and Poling A: Animal olfactory detection of human diseases: Guidelines and systematic review. J Vet Behav 20: 59-73, 2017.

28 Shvartsburg AA, Tang K and Smith RD: Modeling the resolution and sensitivity of FAIMS analyses. J Am Soc Mass Spectrom 15: 1487-1498, 2004.

29 Covington JA, van. der Schee MP, Edge ASL, Boyle B, Savage RS and Arasaradnam RP: The application of FAIMS gas analysis in medical diagnostics. Analyst 140: 6775-6781, 2015.

30 Levin DS, Miller RA, Nazarov EG and Vouros P: Rapid separation and quantitative analysis of peptides using a new nanoelectrospray- differential mobility spectrometer-mass spectrometer system. Analyt Chem 78: 5443-5452, 2006.

31 Silva CL, Passos M and Câmara JS: Investigation of urinary volatile organic metabolites as potential cancer biomarkers by solid-phase microextraction in combination with gas chromatography-mass spectrometry. Br J Cancer 105: 1894-1904, 2011.

32 Venäläinen MK, Roine AN, Häkkinen MR, Vepsäläinen JJ, Kumpulainen PS, Kiviniemi MS, Lehtimäki T, Oksala NK and Rantanen TK: Altered polyamine profiles in colorectal cancer. Anticancer Res 38: 3601-3607, 2018.
33 Phanstiel O: An overview of polyamine metabolism in pancreatic ductal adenocarcinoma. Int J Cancer 142: 1968-1976, 2018.

34 Esfahani S, Sagar N, Kyrou I, Mozdiak E, O'Connell N, Nwokolo $\mathrm{C}$, Bardhan K, Arasaradnam R and Covington J: Variation in gas and volatile compound emissions from human urine as it ages, measured by an electronic nose. Biosensors 6: 4, 2016.

35 Chari ST, Leibson CL, Rabe KG, Timmons LJ, Ransom J, de Andrade $\mathrm{M}$ and Petersen GM: Pancreatic cancer-associated diabetes mellitus: prevalence and temporal association with diagnosis of cancer. Gastroenterology 134: 95-101, 2008.

36 Ghatnekar O, Andersson R, Svensson M, Persson U, Ringdahl $\mathrm{U}$, Zeilon $\mathrm{P}$ and Borrebaeck CAK: Modelling the benefits of early diagnosis of pancreatic cancer using a biomarker signature. Int J Cancer 133: 2392-2397, 2013.

37 Rebours V, Boutron-Ruault MC, Schnee M, Férec C, Maire F, Hammel P, Ruszniewski P and Lévy P: Risk of pancreatic adenocarcinoma in patients with hereditary pancreatitis: A national exhaustive series. Am J Gastroenterol 103: 111-119, 2008.

Received November 27, 2018

Revised December 6, 2018

Accepted December 7, 2018 